\title{
What Is the Evolution of Telemedicine and e-Health in North Africa? A Systematic Review
}

\author{
De la Torre Díez $\mathrm{I}^{1 *}$, Hamrioui $\mathrm{S}^{2}$, Góngora Alonso $\mathrm{s}^{1}$, López-Coronado $\mathrm{M}^{1}$ and Berbey $\mathrm{A}^{3}$ \\ ${ }^{1}$ Department of Signal Theory and Communications \& Telematics Engineering, University of Valladolid, Spain \\ ${ }^{2}$ Bretagne Loire and Nantes Universities, IETR Polytech Nantes, France \\ ${ }^{3}$ Technological University of Panama, Vía Centenario, Panama
}

*Corresponding author: De la Torre Díez I, Department of Signal Theory and Communications \& Telematics Engineering, University of Valladolid, Paseo de Belén, 15. 47011 Valladolid, Spain

submission: October 3, 2018; Published: October 17, 2018

\begin{abstract}
Background: In recent years has been a rapid increase in the use of information technologies in the health sector, tools such as telemedicine and e-Health have been recently incorporated to facilitate access to essential services. Despite their potential benefits - in the North African countries-the experience with these systems has been limited, there have been numerous obstacles, such as doctor shortage and technological barriers.
\end{abstract}

Objective: The main objective of our paper is to show a review of the existing research works in literature regarding the current situation of telemedicine and e-Health in North Africa.

Methods: Several academic databases - such as Science Direct, IEEE Xplore, Web of Science, Google Scholar, Scopus and PubMed - were used to perform searches. The most important search parameter considered was the date of publication, which must be within the last 10 years. The most interesting articles were selected considering the following criteria: Articles in English language with the following keywords: "Telemedicine" AND/OR “eHealth” AND (“Africa” AND/OR “Morocco” AND/OR “Algeria” AND/OR “Tunisia” AND/OR “Libya” AND/OR “Egypt” AND/ OR "Sudan”).

Result: A total of 84 articles were found on telemedicine and eHealth in North Africa of which 25 have been identified as relevant work. Many of the publications found show the existing problems in these countries as well as systems developed in telemedicine and e-Health, being Egypt the country with the highest percentage (35.29\%) and followed by Morocco and Libya with 17.65\%, Tunisia and Algeria with 11.76\% and Sudan with 5.88\%.

Conclusion: From the review of the research articles analyzed it can be said that political and economic issues are a determining factor for the development of telemedicine and e-Health in North Africa, various limitations exist to expand these systems mainly in rural areas. Hence, one main contribution of our paper is to generate new developments that contribute to the management and quality of patient care for improvement.

Keywords: e-Health; North Africa; Review; Telemedicine

\section{Introduction}

The telemedicine term represents the contribution of the new technology that combines knowledge exchange between health professionals and provides patients with access to quality services. Also, the adoption of new technology within a health organization is considered a significant change in the process [1,2].

e-Health is a fairly recent paradigm that leads to a health system new vision, for its improvement in cost reduction terms, decrease of inaccuracies and information quality [3,4]. The main objective of telemedicine $[5,6]$ and its applications is to improve the availability of various medical and health care services despite geographical and economic barriers such as: control of home health [7] guaranteeing that elderly patients can lead an independent life [8] and reducing its direct and indirect costs.
In developed countries-especially in European countries, such as Germany, France, United Kingdom, Norway, Sweden and othersthese information systems are remarkably solid in the medical field, hence most of the hospitals are electronically linked [9].

In case of North African countries - such as: Egypt, Morocco, Algeria, Tunisia, Libya and Sudan - the progress is not so distinguishable, a large percentage of people still lives in rural and peri-urban areas, where there is reduced access and provision of health services, which would benefit from advanced service schemes with architectures and technical support tools [4]. The governments of these developing countries want their country to achieve competitive advantage from investing in information systems programs to help citizens by offering them special and convenient services [9]. 
The reticence to use Information and Communications Technology (ICT) by health professionals, the political and cultural issues and the limited infrastructure resources of these countries are factors that hinder the development of telemedicine and e-Health. Hence, the main objective of our paper is to conduct a review of the state of the art regarding the status of telemedicine and eHealth in North Africa by considering the last 10years (from 2008 to date).

There are similar reviews that base their study on identifying and classifying the diverse applications of health information technologies to assess the current status in developing countries and explore the possibilities for the research, as well as the adequate classification and overview of telemedicine projects in developing countries $[10,11]$. In this paper we aim to present the research work conducted during the last 10 years on telemedicine and e-Health, specifically, in North African countries, and thus being able to establish new challenges in this field and showing the originality and viability of this study.

The methodology used in this review is following described. Afterwards, the results obtained are discussed and the conclusion of this research work is presented.

\section{Methodology}

A review of the published work related to the current situation of telemedicine and e-Health in North African countries was developed, considering the date of publication, which must be within 10years, from 2008 to date. Several systems and academic databases were used, such as Science Direct, IEEE Xplore, Web of Science, Google Scholar, Scopus, PubMed, etc. As part of the search strategy, there were used the following keywords: "Telemedicine" AND/OR "eHealth" AND ("Africa" AND/OR "Morocco" AND/OR "Algeria" AND/OR "Tunisia" AND/OR "Libya" AND/OR "Egypt" AND/OR "Sudan"). There were also considered the abstract, the title and keywords for selecting the most relevant articles regarding the current situation of these countries in telemedicine and e-Health.

Several criteria were considered to identify a document as relevant:

A. Project studies of de telemedicine and e-Health in North African countries.

B. Studies where the main objective is focused on issues that delay the development of telemedicine in these countries.

C. Studies aimed to countries different from North African countries are eliminated.

D. Studies centred on non-specific topics different from telemedicine and e-Health are eliminated.

E. Publications must be written in English.

The selection process of the papers was done by reading the titles and abstracts of the search results obtained; the papers were classified by reading their abstract as well as the full article when required. The Prism methodology [12] has been used to perform the flow diagram shown in Figure 1, which displays the results obtained using the previously described search strategy, as well as the dismissed and duplicated articles.

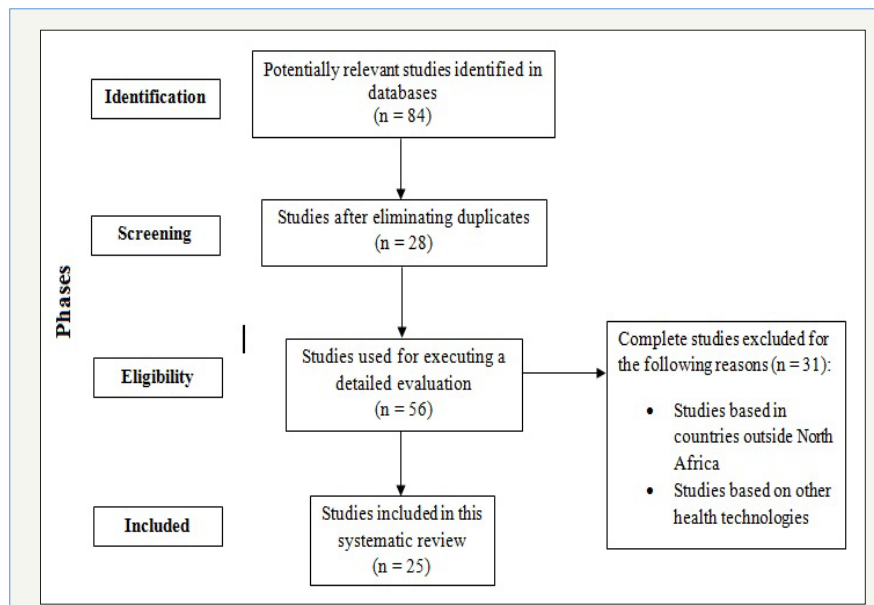

Figure 1: Flow diagram All articles repeated in more than one database were eliminated and filtered by the given criteria, resulting in 25 relevant contributions. The following section are shown the most relevant articles found.

\section{Result}

Currently, many countries have managed to integrate telemedicine [13] and advanced technologies into a wide range of healthcare processes including diagnosis [14], treatment, disease prevention, education and health research. However, many developing countries [15] still cannot maintain significant telemedicine projects. The literature review conducted, and a summary of the results obtained will be explained in the following section.

\section{Literature Review}

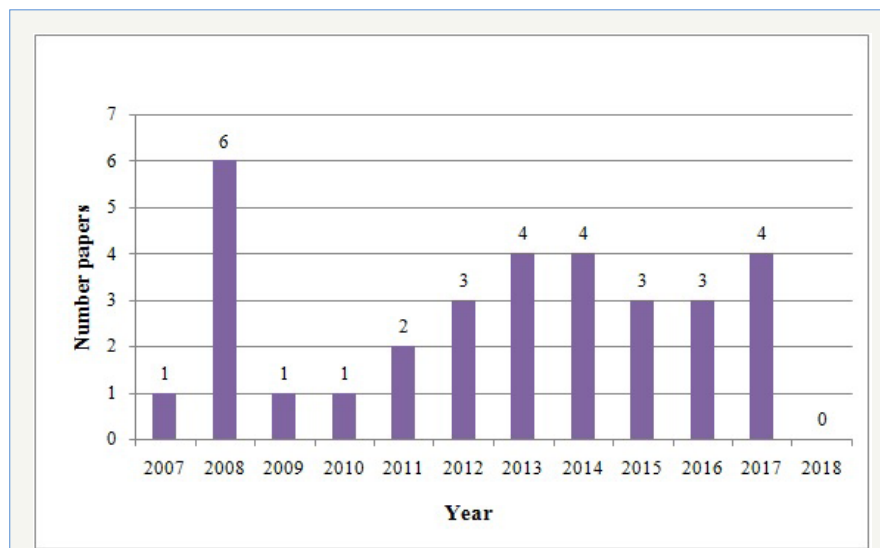

Figure 2: Statistics corresponding to the relevant papers found corresponding to the last 10years.

In the review of the literature we found a series of relevant research papers (Figure 2) corresponding to the last 10 years, which describe the actual situation of North African countries in the telemedicine and e-Health areas. 
According to Nakkas et al. [16], the telecommunications infrastructure in Africa is poor, in part this is owed to the long history of the continent related to the civil unrest and wars, which has delayed economies and infrastructure for up to seven years per each year of unrest. Only 6,7\% of households in Africa have Internet access in their homes, $16,3 \%$ of people use the Internet and the spreading of fixed broadband is $0,3 \%$. The Internet spreading in Africa is half of the one in Asia and the Pacific and is the least spreaded of any developing world regions. The costs of telecommunications in Africa - when expressed as a percentage of per capita, monthly, gross national product - are very high and 14 out of the 20 most expensive countries are in Africa [16]. In rural areas, where the poorest need telemedicine, it is less likely to be provided due to an inadequate infrastructure and high connectivity cost. In addition, there is limited awareness of telemedicine on healthcare professionals and the patient community, as well as lack of action from the government $[16,17]$. The complexity of the African society - with different cultural and religious practices is another factor that reflects the attitude and understanding of people about health issues. As results, most people do not accept the provided health care system model.

In the recent study by Pérez [18] is posed that Africa is the poorest continent with the lowest human development indices worldwide. An African cardiac patient program has been developed as an alternative by Spanish NGDO Recover Foundation, Hospitals for Africa, which includes a telemedicine platform that connects African professionals with Spanish cardiologist for diagnosis collaboration, discussion and assessment of cases with therapeutic purposes. The platform, corresponding to the program of the cardiac patient's group, has a format that allows cases to be uploaded from Africa and professionals from both continents can interact via online. The interface allows to include the medical history, scanned, the diagnostic test files (blood tests, electrocardiograms, echocardiograms, etc.) and a discussion or comments. The latter with simultaneous translation into several languages. As a synchronized platform, it allows access to information from any device, anytime, anywhere, adapting to time availability with only an Internet connection and a user profile [18].

The results obtained in the literature review corresponding to the specific situation of each North African country is following presented.

\section{Egypt}

Hussein \& Khalifa [6] propose that Egypt has achieved significant progress in building an Information Society (IS) by providing an enabling a legal and regulatory framework, and an adequate ICT infrastructure. However, telemedicine projects in Egypt [19] face common problems and challenges that hinder the wide-scale adoption of e-Health systems such as:

A. Lack of patient awareness and acceptance of receiving health services through telemedicine networks and applications.
B. Inability to maintain the functionality of the project due to shortage of financial and legal frameworks.

C. Lack of professionals as well as capacity building programs.

Fouad \& Abdullah [20] propose a telemedicine portal that allows numerous databases to completely interface through Internet. The portal is an innovative solution in the healthcare industry; you can access multiple hospital information systems, inside or outside the hospital environment. One of the most important problems [20] in human health care is the continuous monitoring of patients, especially those that have critical health problems such as heart disease, blood pressure instability, diabetes and others, or even the health state of sports people, hence it is considered of primary interest to Egyptian society.

The study by Tran et al. [21] is one of the first to demonstrate the feasibility of tele dermatology using a combination of newergeneration mobile telephones with specialized software and wireless connectivity in the African continent, specifically in Egypt. It also represents one of the first demonstrations that involves a mobile telephone that transmits both patient history and clinical photographs for diagnosis and receives teleconsultants diagnostic impressions and comments (entirely through cellular connectivity). The performance attained by this study in a developing country suggests that there is a potential for mobile telephones to eliminate one of the most longstanding barriers to the expansion of tele dermatology in one of the most underserved regions of the world [21].

\section{Morocco}

As any other developing country, according to Bennani \& Elayoub [22], "Morocco is facing the challenge of providing accessible, efficient, equitable and quality health services to its population". They pose the various difficulties of the public health sector in Morocco, difficulties that are reflected mainly in terms of low budget allocated to the health sector and structural weaknesses.

In the work by Alsadan et al. [9], results show that besides having access to financial resources, most of the North African countries lagged behind in Health Information Technology (HIT)due to the lack of dedicated financial resources and the existing professional incompetence. Public hospitals financed by governments are lacking the professional use of information technology, while private hospitals do not have sufficient funds to implement telemedicine systems, Electronic Medical Records (EMR) or eHealth.

Le Pape et al. [23] propose that despite progress, health indicators in Morocco are still well below the levels of comparable countries in the region and are highly inequitable, particularly between urban and rural areas. In general, the organization and provision of health care are fragmented and face severe resource limitations. There is no continuous care between ambulatory and inpatient hospital care, and the system suffers from chronic drug 
inventory shortages and a scarcity of human resources across all health personnel categories. Despite these deficiencies, several strategic initiatives have been implemented.

A system was designed in [23] that functions as a patient-centric EMR, an approach designed to facilitate the integration of patient data. A personal health card was proposed, which is independent of the patient's age or insurance coverage, this identifier is a key requirement to guarantee unique medical records and avoid duplicates. Special attention was paid to underserved communities in rural and remote areas [23], whose needs are typically met with medical caravans and mobile units. To better serve these areas, clinical stations preloaded with the patient population's clinical records were suggested and designed to be used either synchronously or asynchronously with the capability to upload data to synchronize server databases.

\section{Libya}

Other factors have contributed to the inhibition of the telemedicine industry so that it does not reach its full global potential [24]. These include the lack of significant reimbursement, licensing problems between states, privacy issues, lack of universal standards and high transmission costs.

El Gatit et al. [24] propose in that health modernization is established as a key objective of the health policy in Libya, and it is important to ensure that health care providers are ready to acknowledge and support the new radical instrumental and administrative changes, which will not only change current medical practice but also professional behavior [25]. Advances in HIT, particularly telemedicine and e-Health, have been considered as key mechanisms by which these changes can be engendered. Directed investment in telemedicine and e-Health promises rapid distribution and deployment of patient-centered information across internal organizational boundaries to global health care. This is shown in the establishment of government agencies specializing in the provision of telemedicine services aiming to connect the main hospitals with rural areas and the establishment of a telemedicine department in the Libyan General Medical Council [24].

\section{Tunisia}

Ilahi \& Ghannouchi [26] propose in that the use of Business Process Management (BPM)in the health field, improving and implementing several relevant commercial processes in telemedicine, such as teleconsultations and remote diagnosis. In this case, teleconsultations process consists of examining a patient or analyzing their data without direct physical interaction through a distant system. Their indications are varied: advice request, diagnosis or therapeutic advice, organization of emergency management with guidance and transfer of the patient to the appropriate service, primary care in a place where there is no doctor (ships, aircraft). The improvement of teleconsultations development is important to the medical decision-making process and consequently, to the quality of health care offered to patients.
Sadok et al. [27] identified a considerable number of difficulties in Tunisia, such as: lack of a political agenda, structural reforms and a coherent investment program, as well as market regulations that affect the telemedicine and e-Health development. One aspect of regional disparities is that the more developed coastal governments, which host a wide variety of economic and financial activities, have a more developed physical infrastructure of broadband networks. Conversely the less developed inner and southern governorates, which have less diversified industrial infrastructure, lack high-speed access networks. Although the average speeds have improved over time the fastest and advantageous packages through fiber optic are destined to satisfy large enterprises and a small group of wealthy customers. The study in [27] has revealed that provision of efficient e-Health services requires a solid broadband infrastructure and imposes different conditions for Quality of Service (QoS).

\section{Algeria and Sudan}

In the review we found only one article from each country that gave us information about what exists in the telemedicine and e-Health systems areas.

The work by Zerroug et al. [28] proposes the project development that provides specialized care in interventional digestive endoscopy throughout Algeria on freelance basis, monitor at distance the clinical parameters and evolution of these patients. This follow-up avoids or diagnoses an outbreak of the disease or the side effects of the medications without the need for hospitalization, when this is not necessary.

The result of this study suggests that regardless of their illiteracy condition, socioeconomic status and age, patients can be telemonitored using mobile telephones [28]. Telemonitoring of chronic diseases is a promising approach in the management of patients, reducing the health care costs.

Mohamed et al. [29] presented in a successful integrated utilization of ICT in the Gezira Family Medicine Project (GFMP)in Sudan. They achieved a high number of telemedicine consultations, with high satisfaction among the candidates. EMRs were introduced in this area for the first time, and more than 165,000 patient files were opened over a period of two years, reflecting the great need for innovative solutions in primary care settings in developing countries.

The GFMP project [29] experience in the use of telemedicine, EMRs and e-learning systems, is rather unique in a developing low-resource country setting. Although the project was politically and financially supported on a national level, the successful implementation was not without barriers and challenges, such as internet coverage and technical problems. The issue of sustainability deserves to be considered and further research is needed to explore the impact of using ICT on health indicators in Sudan.

\section{Summary}

In the review carried out in this research, covering he last 10years, about Telemedicine and e-Health in North African 
countries, we found a small number of investigations that showed the systems development in these countries. In Figure 3, a summary in the form of statistics (Figure 3) from the most relevant research articles is presented.

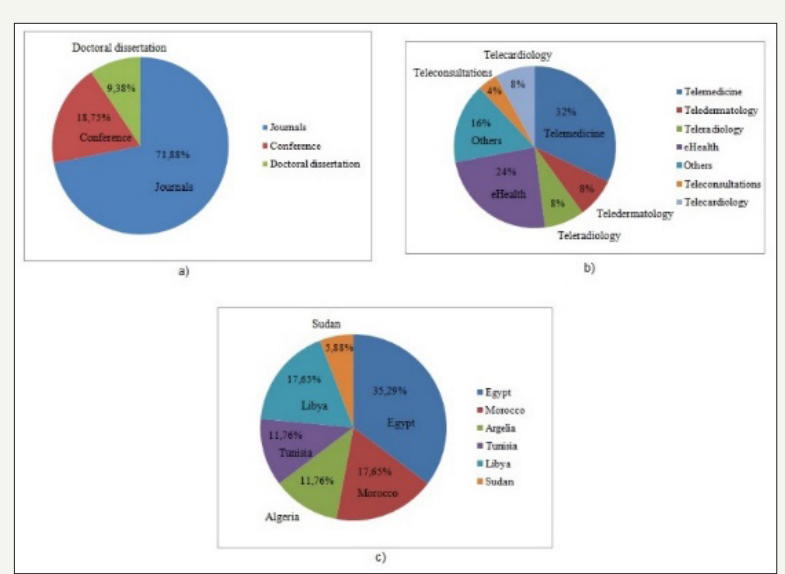

Figure 3: Statistics of the relevant papers identified: a) Percentage of studies found organized by type of publication. b) Percentage of studies organized by Health Information Technology. c) Percentage of studies found organized by country

The largest number of papers found corresponds to journals with $71.88 \%$, followed by conference papers $18.75 \%$ and a smaller number of doctoral dissertations with $9.38 \%$. We included studies that were considered the most relevant in terms of telemedicine and e-Health development in North African countries, also taking into account specialties, such as telecardiology, teledermatology, teleconsultations, teleradiology and others.

\section{Discussion and Conclusion}

The emergence of telemedicine can be seen as an opportunity for national health authorities in North Africa to renew the formulation of medical policies and actions in response to the need of improving health services (such as quality and safety) in rural communities.

In particular, telemedicine creates new clinical risks and responsibilities regarding product and safety problems. Confidentiality and privacy may be hampered by the use of communication devices, which are not controlled by the individual, and may result in a serious invasion of patient privacy. Therefore, there is a need for the rigorous application of the available guidelines and for legal resolution regarding security, quality of health care, privacy and rights.

e-Health tools and apps can provide quick and easy access to EMR when this is needed. They can admit the diagnosis through non-invasive systems based on images. They support surgeons in the planning of clinical interventions using patient-specific digital data and provide access to specialized resources for education and training. Designing and implementing telemedicine solutions, such as mobile services, improve the quality of life of patients suffering from chronic diseases. These solutions also support and optimize their treatment in case of emergency. Wireless technology in e-Health is strongly driven by technological advances and trends [30], such as widespread communications, ubiquitous computing and context awareness.

In summary, the main problem, having an enormous negative impact on telecommunications, in Africa is the high cost of bandwidth and this in turn is affecting the connectivity of an effective electronic network to provide rural telemedicine solutions.

Diversity and variation in socio-economic and cultural situations among the North African countries has caused variation and multiplicity of restrictions in the e-Health implementation. Some of the limitations mentioned in [31] are: The lack of knowledge about the potential benefits of e-health; shortage, and sometimes total lack, of funding by the government and other health care providers; privacy concerns and lack of legal framework; weakness of information infrastructure; complex systems linked to lack of qualified personnel; and lack of data standards that allow exchange of health data in local languages.

The increase in health care costs and aging of the world population contribute to telemedicine network advances for the provision of various health care services. Systems like the health care system presented in [32] can provide applications for diagnostic procedures, chronic diseases maintenance and supervised recovery of a surgical procedure. Patient monitoring applications generally control vital signs and provide feedback and information in real time that help patient recovery.

The results achieved in this review showed us the reduced number of relevant research articles regarding the development of telemedicine and e-Health in North African countries. Hence, a series of future lines have been opened regarding modeling and implementing telemedicine processes in North African countries, such as in Algeria where few publications were found during this review, which could frame the current situation regarding telemedicine and e-Health. Another future line for this research will be related to the design and development of mobile apps for the monitoring of patients with chronic diseases, taking into account the epidemiological realities and the charge disease in the continent.

\section{Acknowledgment}

This research has been made within the Program "Movilidad Investigadores UVA-BANCO SANTANDER 2018", and it has been partially supported by European Commission and the Ministry of Industry, Energy and Tourism under the project AAL-20125036 named "Wetake Care: ICT- based Solution for (Self-) Management of Daily Living".

\section{References}

1. Mezni H, Zeribi BO (2008) Determinants of the individual acceptance of the telemedicine. Proceedings of $3^{\text {rd }}$ International Conference on Information and Communication Technologies: From Theory to Applications, ICTTA, Syria. 
2. Ilahi L, Ghannouchi SA, Martinho R (2014) Healthcare information systems promotion: from an improved management of telemedicine processes to home healthcare processes. Proceedings of the $2^{\text {nd }}$ International Conference on Technological Ecosystems for Enhancing Multiculturality. pp. 333-338.

3. Bennani AE, Belalia M, Oumlil R (2008) As a human factor, the attitude of healthcare practitioners is the primary step for the e-Health: First outcome of an ongoing study in Morocco. Commun IBIMA 3: 506-512.

4. Kamsu FB, Foguem C (2014) Telemedicine and mobile health with integrative medicine in developing countries. Heal Policy Technol 3(4): 264-271.

5. Geissbuhler A, Bagayoko CO, Ly O (2007) The RAFT network: 5years of distance continuing medical education and tele-consultations over the Internet in French-speaking Africa. Int J Med Inform 76(5-6): 351-356.

6. Hussein R, Khalifa A (2012) Telemedicine in Egypt: swot analysis and future trends. GMS Medizinische Inform Biometrie und Epidemiol 8(1): $1-16$.

7. Zarour K (2017) Towards a tele homecare in Algeria: case of diabetes measurement and remote monitoring. Int J E-Health Med Commun 8(4): 61-80.

8. Abo ZM, Ahmed SM, Elnahas O (2014) A wireless emergency telemedicine system for patients monitoring and diagnosis. Int J Telemed Appl. p. 11.

9. Alsadan M, Metwally AEl, Ali A, Jamal A, Khalifa M, et al. (2015) Health Information Technology (HIT) in Arab countries: a systematic review study on hit progress. J Health Inform Dev Ctries 9(2): 32-49.

10. Combi C, Pozzani G, Pozzi G (2016) Telemedicine for developing countries a survey and some design issues. Appl Clin Inform 7(4): 10251050.

11. Liberati A, Altman DG, Tetzlaff J, Mulrow C, Gøtzsche PC, et al. (2009) The PRISMA statement for reporting systematic reviews and metaanalyses of studies that evaluate healthcare interventions: explanation and elaboration. BMJ 339: b2700.

12. Okoroafor IJ, Chukwuneke FN, Ifebunandu N, Onyeka TC, Ekwueme CO, et al. (2017) Telemedicine and biomedical care in Africa: Prospects and challenges. Niger J Clin Pract 20(1): 1-5.

13. Ayad E (2011) Virtual telepathology in Egypt, applications of WSI in Cairo University. Diagn Pathol 6(1): S1

14. Mekawei NM (2013) Factors affecting adoption of e-Health in Egypt. Doctoral dissertation, Middlesex University, UK, p. 251.

15. Mars M (2013) Telemedicine and advances in urban and rural healthcare delivery in Africa. Prog Cardiovasc Dis 56(3): 326-335.

16. Nakkas HA, Scott PJ, Briggs JS (2015) Health information technology in developing countries: a structured literature review with reference to the case of libya. Int J Medical, Heal Biomed Pharm Eng 9(1): 9-18.

17. Iluyemi A, Briggs J, Burger RA (2008) Health service delivery in developing countries through eHealth: making the case for low-cost wireless infrastructures. Proceedings of $5^{\text {th }}$ IET Seminar on Appropriate Healthcare Technologies for Developing Countries, UK, pp. 1-6.

18. Pérez MD (2017) Telemedicine program and cardiac Africans patients treated in Spain. Med Clin (Barc) 148(1): 40-41.

19. Stadelmann J (2012) Assessing readiness for e-Health in Egypt: a case study of Cairo university hospitals. Am Univ Cairo, Egypt.

20. Fouad HA, Abdullah HH (2013) ICT based Telemedicine for the Egyptian Society. Proceedings of $2^{\text {nd }}$ International Conference on Information Technology Convergence and Services, ITCSE, India, pp. 53-64.

21. Tran K, Ayad M, Weinberg J, Cherng A, Chowdhury M, et al. (2011) Mobile tele dermatology in the developing world: Implications of a feasibility study on 30 Egyptian patients with common skin diseases. J Am Acad Dermatol 64(2): 302-309.

22. Bennani AE, Elayoubi M (2008) An insight into e-health programs in Morocco: what is the Realistic Solution? Commun IBIMA 1(2): 228-233.

23. Le Pape MA, Suárez JCN, Mhayi A, Haazen D, Özaltin E (2017) Developing an HMIS architecture framework to support a national health care e-Health strategy reform: a case study from Morocco. Heal Syst Reform 3(1): 56-67.

24. El Gatit AM, Tabet AS, Sherief M, Warieth G, Abougharsa M, et al. (2008) Effects of an awareness symposium on perception of Libyan physicians regarding telemedicine. East Mediterr Heal J 14(4): 926-930.

25. Sharif MEM (2014) Learning readiness assessment framework in promoting e-Health engagement among libyan nurses. Univ Sains Malaysia, Malaysia, p. 1.

26. Ilahi L, Ghannouchi SA (2013) Improving telemedicine processes via bpm. Procedia Technol 9: 1209-1216.

27. Sadok M, Chatta R, Bednar P (2016) ICT for development in Tunisia: Going the last mile. Technol Soc 46: 63-69.

28. Zerroug M, Sari Z (2010) Algeria: Innovative health care solutions enabled by technology development to meet critical needs in remote areas. Mob eHealth Solut Dev Ctries 33.

29. Mohamed KG, Hunskaar S, Abdelrahman SH, Malik EM (2015) Telemedicine and e-learning in a primary care setting in sudan: the experience of the gezira family medicine project. Int J Family Med, p. 7.

30. Khaddar MAE, Harroud H, Boulmalf M, Elkoutbi M, Habbanil A (2012) Emerging wireless technologies in e-Health trends, challenges and framework design issues. Proceedings of International Conference on Multimedia Computing and Systems (ICMCS) pp. 440-445.

31. Al Shorbaji N (2008) e-Health in the eastern Mediterranean region: a decade of challenges and achievements. East Mediterr Heal J 14: 157173.

32. Negra R, Jemili I, Belghith A (2016) A wireless body area networks: applications and technologies. Procedia Comput Sci 83: 1274-1281.
Creative Commons Attribution 4.0

International License

For possible submissions Click Here

\section{Submit Article}

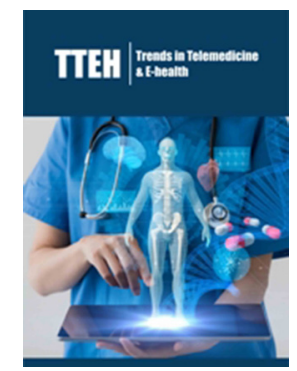

\section{Trends in Telemedicine \& E-health}

\section{Benefits of Publishing with us}

- High-level peer review and editorial services

- Freely accessible online immediately upon publication

- Authors retain the copyright to their work

- Licensing it under a Creative Commons license

- Visibility through different online platforms 